\title{
10000 Transfemoral Carotid Artery Stenting Nechnical Using Proximal Balloon Protection for Patients with Severe Elongation of the Aortic Arch: Inner-catheter Exchange with the Balloon Guide Catheter Anchored ("BGA Exchange")
}

Kei Miyata, ${ }^{1,2}$ Satoshi lihoshi, ${ }^{1}$ Rintaro Yokoyama, ${ }^{1}$ Kentaro Toyama, ${ }^{1}$ Katsuya Komatsu, ${ }^{1}$ Masahiko Wanibuchi, ${ }^{1}$ and Nobuhiro Mikuni ${ }^{1}$

Objective: Anatomical factors such as aortic arch elongation or angulation in elderly patients with arteriosclerotic change often make it difficult to perform carotid artery stenting (CAS) via the transfemoral approach under proximal balloon protection (PBP). We report a novel technique of transfemoral CAS using the inner catheter exchange method for a patient with a type III aortic arch.

Case Presentations: A 78-year-old man presented with symptomatic left carotid artery stenosis. A balloon guide catheter (BGC) was needed for PBP via the transfemoral approach. Initially, the modified Simmons-shaped inner catheter could not follow the half-stiff long guidewire to the external carotid artery (ECA) by the co-axial method. Accordingly, the inner catheter was safely exchanged with a flexible JB2 catheter with the balloon of the BGC fully inflated on the way to the left common carotid artery (CCA). Following the half-stiff long guidewire and with the JB2 catheter advanced into the distal portion of the left ECA beforehand, the BGC was successfully navigated into the left CCA below the bifurcation. Further procedures were performed without any complications.

Conclusion: This technique might be useful in patients with high risk of distal thromboembolic complications in transfemoral CAS with severe elongation of the aortic arch.

Keywords > CAS, transfemoral, proximal balloon protection, type III aortic arch

\section{Introduction}

Aortic arch elongation or bovine arch, which often occurs in elderly persons or in the presence of arteriosclerotic lesions,

${ }^{1}$ Department of Neurosurgery, Sapporo Medical University, Chuo-ku, Sapporo, Japan

${ }^{2}$ Department of Emergency Medicine, Sapporo Medical University, Chuo-ku, Sapporo, Japan

Received: April 11, 2016; Accepted June 28, 2016

Corresponding author: Kei Miyata. Department of Neurosurgery, Sapporo Medical University, South 1 West 16, Chuo-ku, Sapporo 060-8543, Japan

Email: miyata@sapmed.ac.jp

All authors pledge that this manuscript does not contain previously published material and is not under consideration for publication elsewhere.

(C)2016 The Editorial Committee of Journal of Neuroendovascular Therapy. All rights reserved. makes carotid artery stenting (CAS) with a transfemoral approach difficult. ${ }^{1)}$ In such cases, a right brachial artery approach is frequently used, ${ }^{2,3)}$ but some issues still remain to be resolved: ischemic disorder at the puncture site, the difficulty in some patients of catheter insertion into the left common carotid artery (CCA), and the infrequent use of proximal balloon protection $(\mathrm{PBP})^{4}$ ) with a balloon guide catheter (BGC) for the prevention of distal embolism. ${ }^{5)}$ Furthermore, previously reported approach methods such as the concurrent use of a snare wire ${ }^{6,7)}$ and the direct puncture of the superficial temporal artery ${ }^{8)}$ and common carotid artery ${ }^{9,10)}$ are generally hard to adopt.

We performed transfemoral CAS with PBP safely and effectively in a patient with marked stenosis of the left cervical internal carotid artery (ICA) and marked elongation of the aortic arch. Here we report on our procedure, which consists of inner-catheter exchange with an anchored 


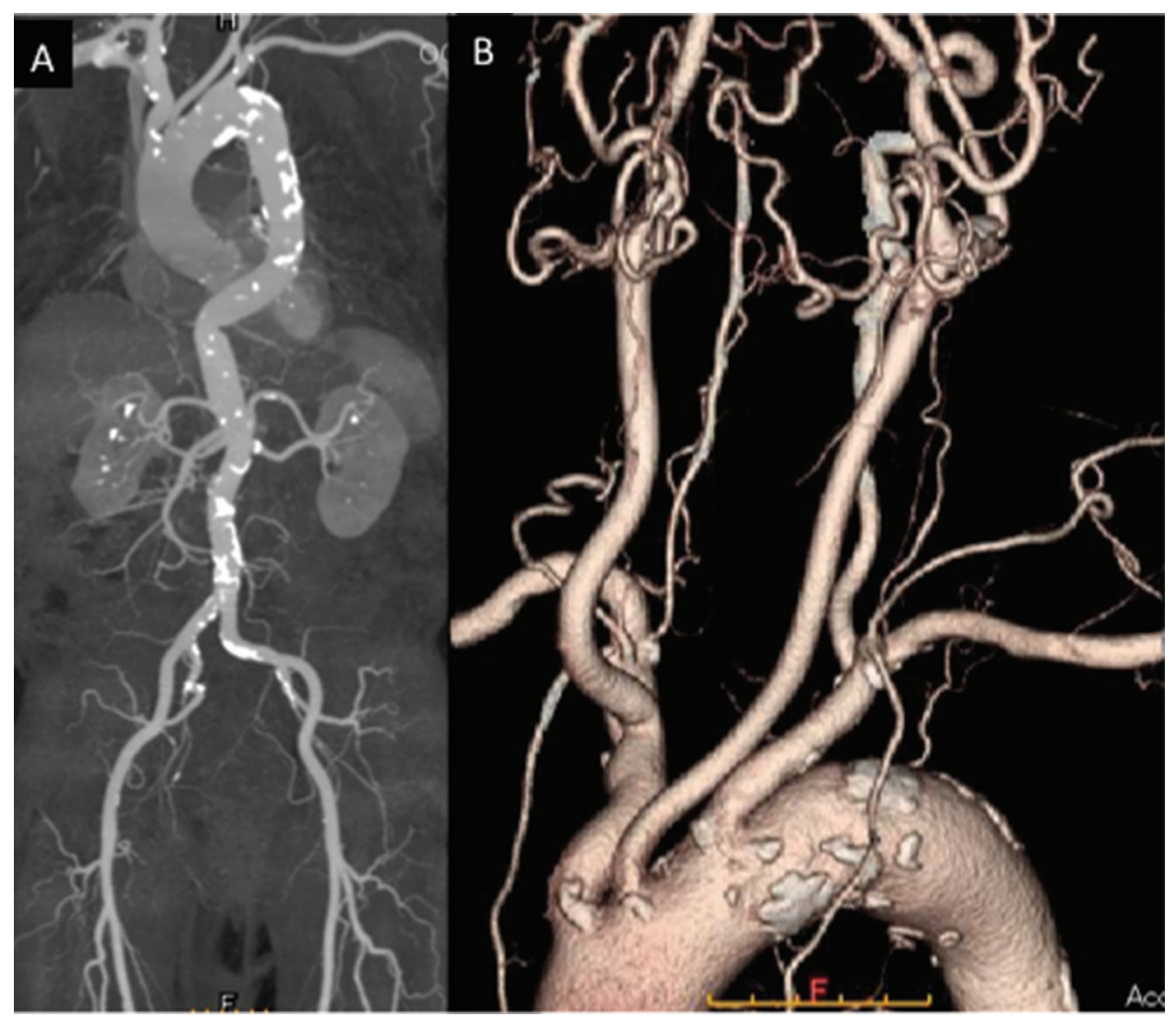

Fig. 1 (A) Maximum intensity projection (MIP) of 3D-CT angiography showed tortuosity and prominent calcification of the descending aorta. (B) Left anterior oblique view of CTA showed prominent calcification of the left internal carotid artery and the origin of the left common carotid artery presenting a type III aortic arch. CTA: computed tomography angiography

balloon guide catheter, and which we call the BGA (Balloon Guide catheter Anchored) exchange method.

\section{Case Presentation}

A 78-year-old male who came to our hospital for a full medical evaluation due to frequent loss of consciousness was diagnosed with marked stenosis of the left cervical ICA (95\% according to the NASCET (The North American Symptomatic Carotid Endarterectomy Trail) method for calculating the degree of stenosis) Diffusion-weighted magnetic resonance imaging (DW-MRI) of the brain revealed a subacute ischemic lesion in the left inferior frontal gyrus. Single photon emission computed tomography (SPECT) of cerebral blood flow showed an approximately $20 \%$ decrease in cerebral blood flow on the affected side relative to the contralateral side. The lesion was determined to be symptomatic, and we concluded that revascularization was indicated. The results of two-dimensional T1 fast-spin-echo (plaque signal intensity relative to adjacent sternocleidomastoid muscle was 1.06) indicated no fragility of carotid artery plaque. Echocardiography showed a decrease in the left ventricular ejection fraction (35\%). Consequently, CAS was planned, though the risk of CEA was also evaluated. 3D-CT angiography revealed tortuosity of the thoracic aorta and calcification of the aortic arch. The arch elongation was graded as aortic arch type III, ${ }^{11)}$ in which the origin of the left CCA branches from the low position of the medial curve of the aortic arch (Figs. 1A and 1B). As these findings suggested that CAS through a transfemoral approach would be difficult, we selected CAS with PBP through a transfemoral approach for the present case because of the high risk of distal embolism related to a distal embolic protection device passing through the symptomatic severe stenotic lesion.

\section{Intravascular treatment}

Clopidogrel $75 \mathrm{mg}$ and aspirin $100 \mathrm{mg}$ were administered daily starting 5 days before the procedure. The procedure was attempted under local anesthesia. An 8 Fr OPTIMO temporary occlusion balloon (Tokai Medical Products, 


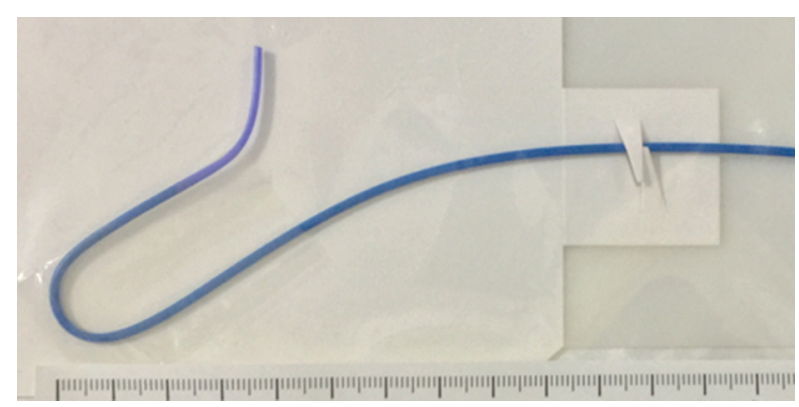

Fig. 2 Tip of the 5.5 Fr Modified Simmons-shaped catheter (SIB; Medikit Co. Ltd., Tokyo).

Aichi) was selected as a BGC, and a 5.5 Fr modified Simmons-shaped catheter (SIB, Medikit, Tokyo) (Fig. 2), characterized by shaft rigidity and strong angle-holding power, was selected as an inner catheter. A catheter introducer (long sheath, $8 \mathrm{Fr}, 40 \mathrm{~cm}$, Medikit) was inserted into the right femoral artery. Initially, we intended to manipulate the SIB catheter into the Simmons shape through ascending aorta operations, but the intra-ventricular aberration of the guidewire and catheter end caused paroxysmal tachycardia and complete atrioventricular block. Accordingly, we manipulated the SIB catheter into the Simmons shape using the crossover technique at the union of the common iliac artery.

The details of the procedure are depicted in Figs. 3A to 3G. A 0.035-inch 300-cm long guidewire (Radifocus Guidewire M standard type, Terumo, Tokyo) and a JB2shaped inner catheter (TEMPO BENTSON II, $5 \mathrm{Fr}, 125 \mathrm{~cm}$, special, Cordis, Cardinal Health Inc., Dublin, OH, U.S.A.) were inserted into the lumen of a BGC and guided to the distal left femoral artery on the contralateral side (Fig. 3A). The JB2-shaped inner catheter was removed while the guidewire was left behind, and the SIB catheter was guided to the left common iliac artery using the exchange method. The SIB catheter was pulled back to the union of the common iliac artery to prepare the Simmons shape (Fig. 3B). Preserving its new shape, the SIB catheter was ascended in the thoracic aorta and inserted into the ostium of the left CCA (Fig. 3C). A half-stiff 0.035 -inch 300-cm long guidewire (Radifocus Guidewire M half stiff type, Terumo, Tokyo) was advanced to the distal external carotid artery (ECA). With traction on the SIB catheter, a BGC with the balloon half-inflated was inserted to the height of the SIB catheter end (the "kite-flying" method; Fig. 3D, arrow). In addition, we attempted to position the SIB catheter at the distal ECA with the balloon maximally inflated to fix the BGC. We were not able to guide it to this point, however, and the BGC kicked back, allowing the system to slide.
We then decided to exchange the SIB catheter for a flexible inner catheter. The details of the method by which the inner catheter was exchanged while the balloon guide catheter was anchored (BGA exchange method) are provided below. To prevent the BGC from sliding, its balloon was maximally inflated. With the long guidewire left in place, the SIB catheter was carefully removed from the lumen of the BGC (Fig. 3E, dotted arrow). Subsequently, a JB2-shaped inner catheter was inserted using the exchange method, and guided to the distal ECA (Fig. 3F, arrow). Finally, the BGC was advanced to a point just proximal to the stenosis with the balloon deflated (Fig. 3G, arrow head). The balloon was maximally inflated at the left CCA during a total of $3 \mathrm{~min}$.

Subsequently, a balloon catheter (Percusurge Guard Wire System $300 \mathrm{~cm}$ : Covidien, Minneapolis, MN, U.S.A.) was carefully moved across the stenotic lesion and positioned at the high cervical ICA under proximal balloon occlusion. Then the distal balloon catheter was also inflated so that double balloon protection was achieved. Predilation was performed with a percutaneous transluminal angioplasty balloon (Sterling Monorail Balloon Catheter $3.0 \mathrm{~mm} \times 40 \mathrm{~mm}$ : Boston Scientific, Marlborough, MA, U.S.A.). A tapered stent (Protege RX Carotid Stent System tapered 8-6 mm $\times$ $40 \mathrm{~mm}$ : Medtronic, Dublin, Ireland) was deployed, and postdilation was performed using a Sterling Balloon Catheter $4.0 \mathrm{~mm} \times 30 \mathrm{~mm}$ (Boston Scientific). Manual aspiration of a total of approximately $160 \mathrm{ml}$ of blood was performed using the aspiration catheter. The proximal and distal balloons were deflated in sequence, and the CAS procedure was finished without complications. Postoperative angiography (Fig. 4B) showed effective dilation (operation time: $1 \mathrm{~h}$ and $55 \mathrm{~min}$, total blood flow blockage time: $18 \mathrm{~min}$, total dose of heparin: 5000 units, maximum ACT: 310 seconds). DW-MRI the day after the procedure revealed no new ischemic foci.

\section{Discussion}

The "BGA exchange" method contributed to successful outcome of CAS under PBP in a patient with carotid artery stenosis in whom the shape of the aortic arch made a transfemoral approach difficult. According to a previous study, ${ }^{12}$ a long distance from the orifice of a target blood vessel to the origin of the descending aorta was a predictive indicator of technical difficulty. In the present case, the horizontal distance from the left CCA orifice to the top of the aortic arch was long, and the left CCA branched from the low position of the aortic arch at a small angle. In addition, the patient's type III aortic arch was complicated by calcification, and 

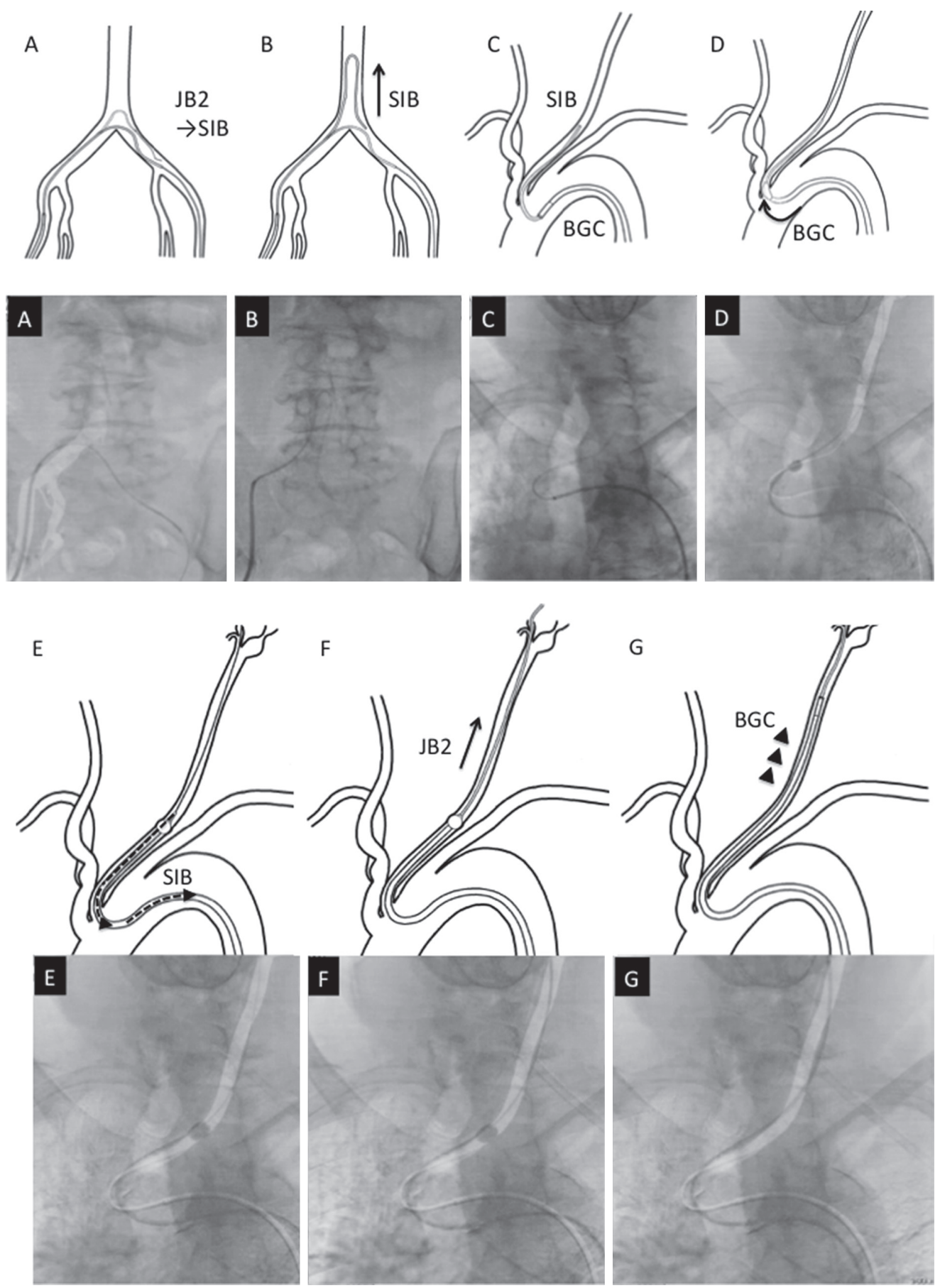

Fig. 3 Schematic drawings and fluoroscopic images depicting the method of inner-catheter exchange with the balloon guide catheter anchored (the BGA Exchange method). (A) The 0.035 -inch long guidewire and the JB2 catheter were advanced into the contralateral left femoral artery. (B) The JB2 catheter was exchanged with a modified Simmons-shaped "SIB" catheter over the long guidewire. The SIB catheter was pulled back to the union of the common iliac artery, and shaped into a Simmons angle (arrow). (C) The SIB catheter was advanced through the descending aorta while the Simmons angle was maintained, and was introduced into the origin of the common carotid artery (CCA). (D) The half-stiff 0.035 -inch long guidewire was inserted into the distal portion of the external carotid artery (ECA). The balloon guide catheter (BGC) was advanced up to the level of the SIB catheter tip with the balloon half-inflated (the "kite-flying" method; arrow). (E) To prevent the BGC from slipping down, the balloon was fully inflated and anchored to the left CCA. The SIB catheter was carefully extracted from the lumen of the BGC (dot-arrow). (F) Over the long half-stiff wire, the JB2 catheter was advanced into the distal ECA (arrow). (G) The BGC was positioned in the left common carotid artery below the bifurcation with the balloon of the BGC deflated (arrowhead). 


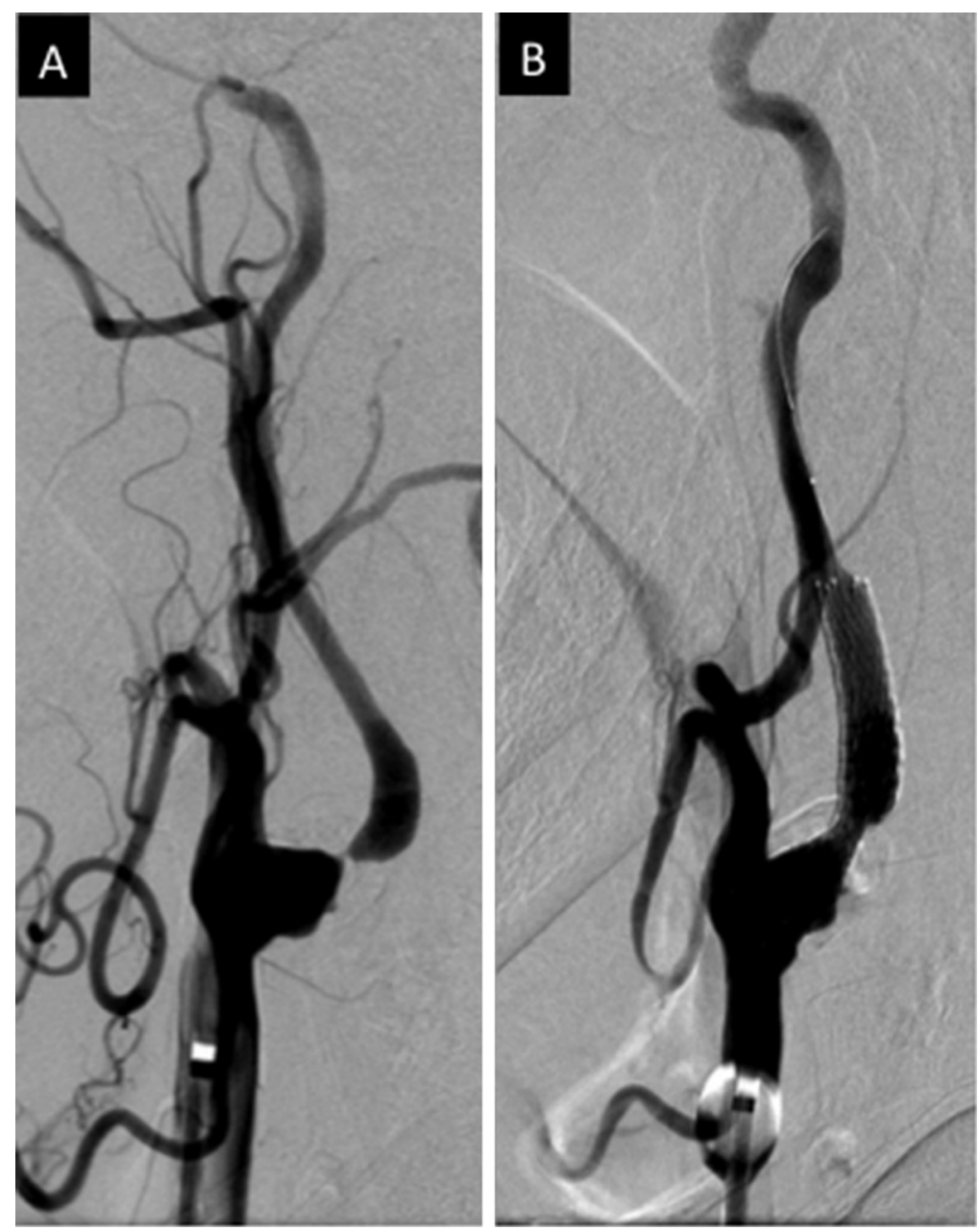

Fig. 4 (A) Preoperative left common carotid angiogram (lateral view) showed 95\% stenosis of the left internal carotid artery (ICA). (B) Postoperative left common carotid angiogram (lateral view) showed adequate dilatation of the left ICA.

several anatomical characteristics made a transfemoral approach difficult. Therefore, the BGA exchange method, which we believe may be useful in future cases, was required to guide the catheter.

This procedure has two distinctive characteristics: (1) the necessity of two inner catheters differing in shape, and (2) the need for just the right degree of balloon inflation when the $\mathrm{BGC}$ is being positioned.

(1) In this procedure, the BGC is safely introduced into the distal CCA by alternately using two inner catheters markedly differing in shape. Initially, the BGC is guided beyond a flexion involving the aortic arch and left CCA origin using a modified Simmons-shaped SIB catheter with shaft rigidity and a half-stiff long guidewire inserted into the distal ECA as axes. If another Simmons-shaped catheter with less shaft rigidity had been attempted, the flexion shape of its end may easily have opened, allowing the guidewire to slide into the ascending aorta. Although the shaft rigidity of the SIB catheter is necessary at this point in the procedure, however, a more flexible inner catheter is needed later to permit distal access. Thus the SIB catheter was exchanged for a flexible JB2 inner catheter, which we used to advance the BGC to the point just proximal to the stenosis.

(2) To advance the BGC and inner catheter to the distal $\mathrm{CCA}$, the balloon had to be inflated to the right degree. Initially, the BGC was advanced up to the level of the SIB catheter tip with the balloon half-inflated according to the "kite-flying" method. Later, the balloon was fully inflated and anchored to the proximal CCA in accordance with the BGA exchange method. Inflating the balloon stabilized the BGC on SIB removal, preventing the system from sliding into the aortic arch. Furthermore, it provided strong support which contributed substantially to the stable insertion of the JB2 inner catheter into the ECA. This method is 
suggested to be effective in patients with severe aortic arch elongation, which makes the transfemoral approach difficult, and in those with left carotid artery lesions, which make a right brachial approach difficult. In addition, it may be applied in the stent-retriever mechanical thrombectomy techniques for acute ischemic stroke, in which BGCs also play a role.

In this procedure, inner-catheter exchange and balloon inflation must be performed while the BGC is positioned; in this point it differs from the standard CAS procedure. The following points should be considered: initially, physicians should try to guide the BGC to the CCA without exchanging the inner catheter. For example, a Simmons-shaped catheter should be guided to the ECA after the balloon is inflated and the BGC is anchored. In patients who cannot tolerate CCA occlusion, the duration of balloon inflation should be shortened. In some patients with CCA diameter $\geq 12 \mathrm{~mm}$, anchoring is impossible. If the branching angle of the aortic arch is large, BGC kinking may make balloon operations or device passage difficult. When a 9 Fr BGC is used, a triple axial system with 4 Fr Simmons-shaped catheters and 6 Fr flexible catheters may be useful for introducing the BGC. This procedure cannot be performed if the Simmons shape cannot be prepared, if the SIB end cannot be inserted to the ostium of the CCA, or if a stiff- or halfstiff-type 0.035-inch guidewire cannot be advanced to the ECA. Careless catheter operations in aortic arches with marked calcification or arteriosclerotic changes cause many complications. ${ }^{1)}$ Increasing the number of options for access may help increase the rate of positive outcome.

\section{Conclusion}

We safely performed CAS under PBP through a transfemoral approach for a stenotic lesion of the carotid artery with marked aortic arch elongation. A BGC was useful for preventing PBP-related distal embolism and guiding the catheter to a lesion which was otherwise difficult to access.

\section{Disclosure Statement}

There is no conflict of interest to be disclosed regarding this article.

\section{References}

1) Lam RC, Lin SC, DeRubertis B, et al: The impact of increasing age on anatomic factors affecting carotid angioplasty and stenting. J Vasc Surg 2007; 45: 875-880.

2) Gonzalez LF, Albuquerque FC, McDougall C: Neurointerventional techniques: tricks of the trade. Brachial and Radial Artery Access. New York: Thieme Medical Publishers, 2015; pp. 16-19.

3) Dahm JB, van Buuren F, Hansen C, et al: The concept of an anatomy related individual arterial access: lowering technical and clinical complications with transradial access in bovineand type-III aortic arch carotid artery stenting. VASA 2011; 40: 468-473.

4) Stabile E, Sannino A, Schiattarella GG, et al: Cerebral embolic lesions detected with diffusion-weighted magnetic resonance imaging following carotid artery stenting: a meta-analysis of 8 studies comparing filter cerebral protection and proximal balloon occlusion. JACC Cardiovasc Interv 2014; 7: 1177-1183.

5) Koge J, Nakahara I, Ohta T, et al: Carotid artery stenting under proximal balloon protection via the transbrachial approach using a balloon guiding catheter: sheathless method with $9 \mathrm{Fr}$ Optimo. JNET 2015; 9: 108-114.

6) Uda T, Murata K, Ichinose T, et al: [Usefulness of the goose neck snare for holding the guiding catheter for carotid artery stenting]. No Shinkei Geka 2007; 35: 673-676. (in Japanese)

7) Ventoruzzo G, Biondi-Zoccai G, Maioli F, et al: A tailored approach to overcoming challenges of a bovine aortic arch during left internal carotid artery stenting. $J$ Endovasc Ther 2012; 19: 329-338.

8) Mitsuhashi Y, Nishio A, Kawakami T, et al: New pull-through technique using the superficial temporal artery for transbrachial carotid artery stenting. Neurol Med Chir (Tokyo) 2009; 49: $320-324$.

9) Bergeron P: Direct percutaneous carotid access for carotid angioplasty and stenting. J Endovasc Ther 2015; 22: 135-138.

10) Matsuda $Y$, Terada $T$, Masuo $O$, et al: The clinical results of transcervical carotid artery stenting and frequency chosen as the approach route of carotid artery stenting in 1,067 consecutive cases. Acta Neurochir (Wien) 2013; 155: 1575-1581.

11) Lin SC, Trocciola SM, Rhee J, et al: Analysis of anatomic factors and age in patients undergoing carotid angioplasty and stenting. Ann Vasc Surg 2005; 19: 798-804.

12) Madhwal S, Rajagopal V, Bhatt DL, et al: Predictors of difficult carotid stenting as determined by aortic arch angiography. J Invasive Cardiol 2008; 20: 200-204. 